\title{
A COEFFICIENT INEQUALITY FOR THE CLASS OF ANALYTIC FUNCTIONS IN THE UNIT DISC
}

\section{YAȘAR POLATOǦLU and METIN BOLCAL}

\author{
Received 31 August 2002
}

The aim of this paper is to give a coefficient inequality for the class of analytic functions in the unit disc $D=\{z|| z \mid<1\}$.

2000 Mathematics Subject Classification: 30C45.

1. Introduction. Let $\Omega$ be the family of functions $\omega(z)$ regular in the disc $D$ and satisfying the conditions $\omega(0)=0$ and $|\omega(z)|<1$ for $z \in D$.

Next, for arbitrary fixed numbers $A$ and $B,-1<A \leq 1,-1 \leq B<A$, denote by $P(A, B)$ the family of functions

$$
p(z)=1+p_{1} z+p_{2} z^{2}+\cdots
$$

regular in $D$ such that $p(z)$ is in $P(A, B)$ if and only if

$$
p(z)=\frac{1+A \omega(z)}{1+B \omega(z)}
$$

for some function $\omega(z) \in \Omega$ and every $z \in D$. The class $P(A, B)$ was introduced by Janowski [3].

Moreover, let $S^{*}(A, B, b)(b \neq 0$, complex) denote the family of functions

$$
f(z)=z+a_{2} z^{2}+\cdots+a_{n} z^{n}+\cdots
$$

regular in $D$ and such that $f(z)$ is in $S^{*}(A, B, b)$ if and only if

$$
1+\frac{1}{b}\left(z \frac{f^{\prime}(z)}{f(z)}-1\right)=p(z)
$$

for some $p(z)$ in $P(A, B)$ and all $z$ in $D$.

For the aim of this paper we need Jack's lemma [2]. "Let $\omega(z)$ be a regular in the unit disc with $\omega(0)=0$, then if $|\omega(z)|$ attains its maximum value on the circle $|z|=r$ at a point $z_{1}$, we can write $z_{1} \omega^{\prime}\left(z_{1}\right)=k \omega\left(z_{1}\right)$, where $k$ is real and $k \geq 1$." 
2. Coefficient inequality. The main purpose of this paper is to give sharp upper bound of the modulus of the coefficient $a_{n}$. Therefore, we need the following lemma.

LEMMA 2.1. The necessary and sufficient condition for $g(z)=z+a_{2} z^{2}+\cdots$ belongs to $S^{*}(A, B, b)$ is

$$
g(z) \in S^{*}(A, B, b) \Longleftrightarrow g(z)= \begin{cases}z \cdot(1+B \omega(z))^{b(A-B) / B}, & B \neq 0, \\ z \cdot e^{b A \omega(z)}, & B=0,\end{cases}
$$

where $\omega(z) \in \Omega$.

PROof. The proof of this lemma is in four steps.

STEP 1. Let $B \neq 0$ and

$$
g(z)=z \cdot(1+B \omega(z))^{b(A-B) / B}
$$

If we take the logarithmic derivative from equality (2.2), we obtain

$$
\frac{1}{b}\left(z \cdot \frac{g^{\prime}(z)}{g(z)}-1\right)=(A-B) \frac{z \cdot \omega^{\prime}(z)}{1+B \omega(z)}
$$

If we use Jack's lemma [2] in equality (2.3), we get

$$
\frac{1}{b}\left(z \frac{g^{\prime}(z)}{g(z)}-1\right)=\frac{(A-B) \omega(z)}{1+B \omega(z)}
$$

After the simple calculations from (2.4), we see that

$$
1+\frac{1}{b}\left(z \cdot \frac{g^{\prime}(z)}{g(z)}-1\right)=\frac{1+A \omega(z)}{1+B \omega(z)}
$$

Equality (2.5) shows that $g(z) \in S^{*}(A, B, b)$.

STEP 2. Let $B=0$ and

$$
g(z)=z \cdot e^{b A \omega(z)}
$$

Similarly, we obtain

$$
1+\frac{1}{b}\left(z \frac{g^{\prime}(z)}{g(z)}-1\right)=\frac{1+A \omega(z)}{1+B \omega(z)}=1+A \omega(z) .
$$

This shows that $g(z) \in S^{*}(A, B, b)$. 
STEP 3. Let $g(z) \in S^{*}(A, B, b)$ and $B \neq 0$, then we have

$$
1+\frac{1}{b}\left(z \frac{g^{\prime}(z)}{g(z)}-1\right)=\frac{1+A \omega(z)}{1+B \omega(z)} .
$$

Equality (2.8) can be written in the from

$$
\frac{g^{\prime}(z)}{g(z)}=\frac{b(A-B)(\omega(z) / z)}{1+B \omega(z)}+\frac{1}{z}
$$

If we use Jack's lemma (2.9), we obtain

$$
\frac{g^{\prime}(z)}{g(z)}=\frac{b(A-B) \omega^{\prime}(z)}{1+B \omega(z)}+\frac{1}{z} .
$$

Integrating both sides of equality (2.10), we get

$$
g(z)=z \cdot(1+B \omega(z))^{b(A-B) / B} .
$$

STEP 4. Let $g(z) \in S^{*}(A, B, b)$ and $B=0$. Similarly, we obtain

$$
g(z)=z \cdot e^{b A \omega(z)}
$$

which ends the proof.

We note that we choose the branch of $(1+B w(z))^{b(A-B) / B}$ such that

$$
(1+B w(0))^{b(A-B) / B}=1 \quad \text { at } z=0 .
$$

THEOREM 2.2. If $f(z)=z+a_{2} z^{2}+\cdots+a_{n} z^{n}+\cdots$ belongs to $S^{*}(A, B, b)$, then

$$
\begin{aligned}
& \left|a_{n}\right| \leq \prod_{k=0}^{n-2} \frac{|b(A-B)+k B|}{k+1} \quad \text { if } B \neq 0, \\
& \left|a_{n}\right| \leq \prod_{k=0}^{n-2} \frac{|b A|}{k+1} \quad \text { if } B=0 .
\end{aligned}
$$

These bounds are sharp because the extremal function is

$$
f_{*}(z)= \begin{cases}\frac{z}{(1-B \delta z)^{-b(A-B) / B}}, & |\delta|=1, \text { if } B \neq 0, \\ z e^{b A z}, & \text { if } B=0 .\end{cases}
$$

Proof. Let $B \neq 0$. If we use the definition of the class $S^{*}(A, B, b)$, then we write

$$
1+\frac{1}{b}\left(z \frac{f^{\prime}(z)}{f(z)}-1\right)=p(z)
$$


Equality (2.16) can be written by using the Taylor expansion of $f(z)$ and $p(z)$ in the form

$$
\begin{aligned}
& z+2 a_{2} z^{2}+3 a_{3} z^{3}+\cdots+n a_{n} z^{n}+\cdots \\
& \quad=\left(z+a_{2} z^{2}+\cdots+a_{n} z^{n}+\cdots\right)\left(1+b p_{1} z+b p_{2} z^{2}+\cdots+b p_{n} z^{n}+\cdots\right) .
\end{aligned}
$$

Evaluating the coefficient of $z^{n}$ in both sides of (2.17), we get

$$
n a_{n}=a_{n}+b p_{1} a_{n-1}+b p_{2} a_{n-2}+\cdots+b p_{n-1} .
$$

on the other hand,

$$
\left|p_{n}\right| \leq(A-B)
$$

Inequality (2.19) was proved by Aouf [1]. If we consider the relations (2.18) and (2.19) together, then we obtain

$$
(n-1)\left|a_{n}\right| \leq|b||A-B|\left(1+\left|a_{2}\right|+\left|a_{3}\right|+\cdots+\left|a_{n-1}\right|\right),
$$

which can be written in the form

$$
\left|a_{n}\right| \leq \frac{1}{(n-1)} \sum_{k=1}^{n-1}|b||A-B|\left|a_{k}\right|, \quad\left|a_{1}\right|=1 .
$$

To prove (2.14), we will use the induction principle.

Now, we consider inequalities (2.21) and

$$
\left|a_{n}\right| \leq \prod_{k=0}^{n-2} \frac{|b(A-B)+k B|}{k+1}
$$

The right-hand sides of these inequalities are the same because

(i) for $n=2$,

$$
\begin{aligned}
& \left|a_{n}\right| \leq \frac{|b||A-B|}{(n-1)} \sum_{k=1}^{n-1}\left|a_{k}\right|, \quad\left|a_{1}\right|=1 \Longrightarrow\left|a_{2}\right| \leq|b||A-B|, \\
& \left|a_{n}\right| \leq \prod_{k=0}^{n-2} \frac{|b(A-B)+k B|}{k+1}=|b(A-B)| \Longrightarrow\left|a_{2}\right| \leq|b||A-B| ;
\end{aligned}
$$


(ii) for $n=3$,

$$
\begin{aligned}
& \left|a_{3}\right| \leq \frac{|b||A-B|}{(n-1)} \sum_{k=1}^{n-1}\left|a_{k}\right|=\frac{1}{2}|b||A-B|\left(1+\left|a_{2}\right|\right) \\
& \Longrightarrow\left|a_{3}\right| \leq \frac{1}{2}|b|^{2}|A-B|^{2}+\frac{1}{2}|b||A-B|, \\
& \left|a_{3}\right| \leq \prod_{k=0}^{n-2} \frac{|b(A-B)+k B|}{k+1}=|b||A-B| \frac{|b(A-B)+B|}{2} \\
& \Longrightarrow\left|a_{3}\right| \leq \frac{1}{2}|b||A-B|[|b||A-B|+|B|] \leq \frac{1}{2}|b||A-B|[|b||A-B|+1] \\
& \Longrightarrow\left|a_{3}\right| \leq \frac{1}{2}|b|^{2}|A-B|^{2}+\frac{1}{2}|b||A+B| .
\end{aligned}
$$

Suppose that this result is true for $n=p$, then we have

$$
\begin{aligned}
& \left|a_{n}\right| \leq \frac{|b||A-B|}{(n-1)} \sum_{k=1}^{n-1}\left|a_{k}\right|, \\
& \left|a_{1}\right|=1 \Longrightarrow\left|a_{p}\right| \leq \frac{|b||A-B|}{(p-1)}\left(1+\left|a_{2}\right|+\left|a_{3}\right|+\cdots+\left|a_{p-1}\right|\right), \\
& \left|a_{n}\right| \leq \prod_{k=0}^{n-2} \frac{|b(A-B)+k B|}{k+1} \\
& \Longrightarrow\left|a_{p}\right| \leq \prod_{k=0}^{p-2} \frac{|b(A-B)+k B|}{k+1} \\
& \Longrightarrow\left|a_{p}\right| \leq \frac{1}{(p-1) !}|b||A-B|(|b||A-B|+1)(|b||A-B|+2) \\
& \cdot(|b||A-B|+3) \cdots(|b||A-B|+(p-2))
\end{aligned}
$$

from (2.25), (2.26), and induction hypothesis, we have

$$
\begin{aligned}
& \frac{|b||A-B|}{(p-1)}\left(1+\left|a_{2}\right|+\left|a_{3}\right|+\cdots+\left|a_{p-1}\right|\right) \\
& =\frac{1}{(p-1) !}|b||A-B|(|b||A-B|+1) \\
& \quad \cdot(|b||A-B|+2) \cdots(|b||A-B|+(p-2)) .
\end{aligned}
$$

If we write $x=|b||A-B|>0$, equality (2.27) can be written in the form.

$$
\begin{aligned}
& \frac{x}{(p-1)}\left(1+\left|a_{2}\right|+\left|a_{3}\right|+\cdots+\left|a_{p-1}\right|\right) \\
& \quad=\frac{1}{(p-1) !} x(x+1)(x+2) \cdots(x+(p-2)) .
\end{aligned}
$$


After the simple calculation from equality (2.28), we get

$$
\begin{aligned}
\frac{1}{p}(x+ & (p-1)) \frac{1}{(p-1)}\left(1+\left|a_{2}\right|+\left|a_{3}\right|+\cdots+\left|a_{p-1}\right|\right) \\
= & \frac{1}{p !}(x+1)(x+2)(x+3) \cdots(x+(p-2))(x+(p-1)) \\
\Longrightarrow & \frac{1}{p}\left[\frac{x}{p-1}\left(1+\left|a_{2}\right|+\left|a_{3}\right|+\cdots+\left|a_{p-1}\right|\right)\right] \\
& +\left[\frac{1}{p}\left(1+\left|a_{2}\right|+\left|a_{3}\right|+\cdots+\left|a_{p-1}\right|\right)\right] \\
= & \frac{1}{p !}(x+1)(x+2)(x+3) \cdots(x+(p-2))(x+(p-1)) \\
\Longrightarrow & \frac{1}{p}\left|a_{p}\right|+\left[\frac{1}{p}\left(1+\left|a_{2}\right|+\left|a_{3}\right|+\cdots+\left|a_{p-1}\right|\right)\right] \\
= & \frac{1}{p !}(x+1)(x+2)(x+3) \cdots(x+(p-2))(x+(p-1)) \\
\Longrightarrow & \frac{x}{p}\left(1+\left|a_{2}\right|+\left|a_{3}\right|+\cdots+\left|a_{p-1}\right|+\left|a_{p}\right|\right) \\
= & \frac{1}{p !} x(x+1)(x+2)(x+3) \cdots(x+(p-2))(x+(p-1)) .
\end{aligned}
$$

Equality (2.29) shows that the result is valid for $n=p+1$.

Therefore, we have (2.14).

COROLLARY 2.3. The first inequality of (2.14) can be rewritten in the form

$$
\begin{aligned}
\left|a_{n}\right| \leq & \prod_{k=0}^{n-2} \frac{|b(A-B)+k b|}{k+1} \\
= & |B(A-B)| \frac{1}{2}|b(A-B)+B| \\
& \cdot \frac{1}{3}|b(A-B)+2 B| \cdots \frac{1}{(n-1)}|b(A-B)+(n-2) B| \\
= & \frac{1}{(n-1) !}|b(A-B)| \cdot|b(A-B)+B| \\
& \cdot|b(A-B)+2 B| \cdots|b(A-B)+(n-2) B| \\
\leq & \frac{1}{(n-1) !}|b(A-B)|(|b(A-B)|+|B|) \\
& \cdot(|b(A-B)|+2|B|) \cdots(|b(A-B)|+(n-2)|B|) .
\end{aligned}
$$

If $A=1, B=-1$, and $b=1$, then

$$
\left|a_{n}\right| \leq \frac{1}{(n-1) !} 2 \cdot(2+1) \cdot(2+2) \cdots n=\frac{n !}{(n-1) !}=n
$$

This is the coefficient inequality for the starlike function which is well known. 
COROLlary 2.4. If $A=1, B=-1$,

$$
\left|a_{n}\right|<\frac{1}{(n-1) !} \prod_{k=0}^{n-2}|2 b+k| .
$$

This inequality was obtained by Aouf [1].

Therefore, by giving the special value to $A, B$, and $b$, we obtain the coefficient inequality for the classes $S^{*}(1,-1, \beta), S^{*}\left(1,-1, e^{-i \lambda} \operatorname{Cos} \lambda\right), S^{*}(1,-1,(1-$ $\left.\beta) e^{-i \lambda} \operatorname{Cos} \lambda\right), S^{*}(1,0, b), S^{*}(\beta, 0, b), S^{*}(\beta,-\beta, b), S^{*}(1,(-1+1 / M), b)$, and $S^{*}(1-2 \beta,-1, b)$, where $0 \leq \beta<1,|\lambda|<\pi / 2$, and $M>1$.

\section{REFERENCES}

[1] M. K. Aouf, On a class of p-valent starlike functions of order $\alpha$, Int. J. Math. Math. Sci. 10 (1987), no. 4, 733-744.

[2] I. S. Jack, Functions starlike and convex of order $\alpha$, J. London Math. Soc. (2) 3 (1971), 469-474.

[3] W. Janowski, Some extremal problems for certain families of analytic functions. I, Ann. Polon. Math. 28 (1973), 297-326.

Yaşar Polatoğlu: Department of Mathematics and Computer Science, Faculty of Science and Letters, Istanbul Kültür University, Sirinevler 34510, Istanbul, Turkey

E-mail address: y. polatoglu@iku.edu.tr

Metin Bolcal: Department of Mathematics and Computer Science, Faculty of Science and Letters, Istanbul Kültür University, Sirinevler 34510, Istanbul, Turkey

E-mail address: m. bolcal@iku. edu.tr 


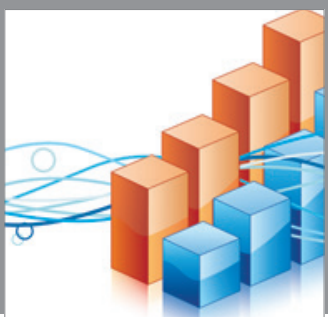

Advances in

Operations Research

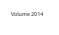

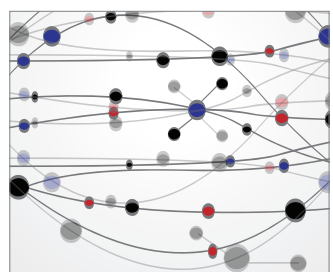

\section{The Scientific} World Journal
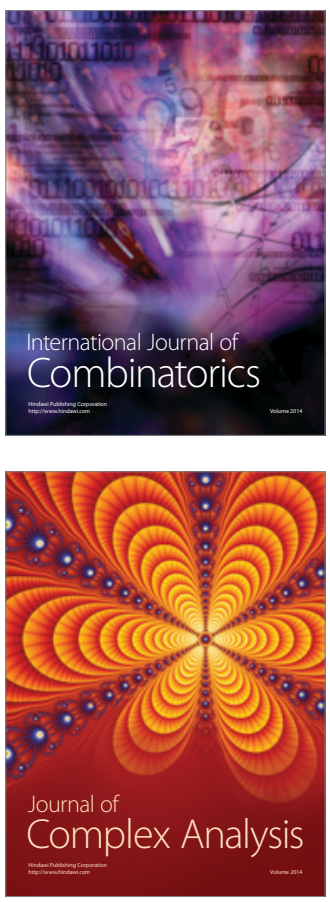

International Journal of

Mathematics and

Mathematical

Sciences
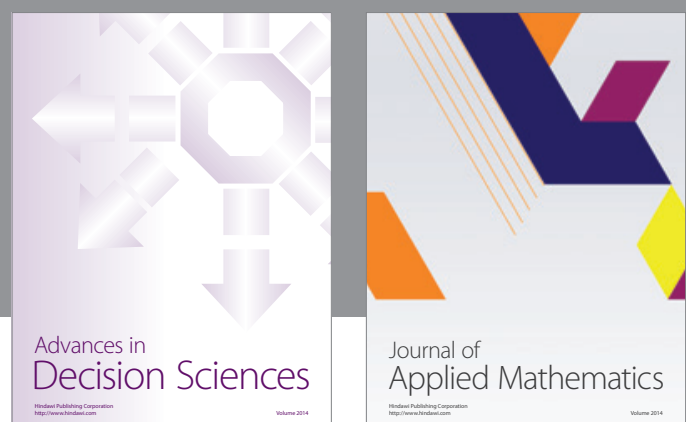

Journal of

Applied Mathematics
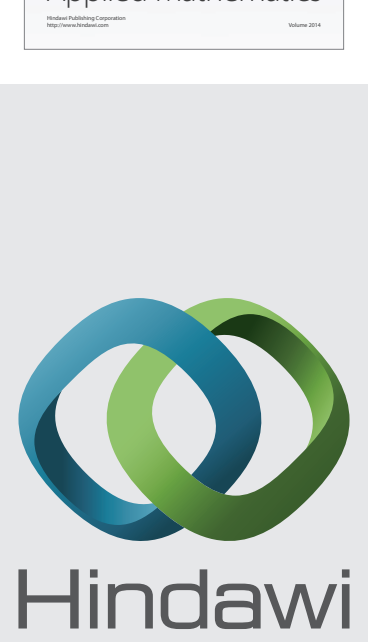

Submit your manuscripts at http://www.hindawi.com
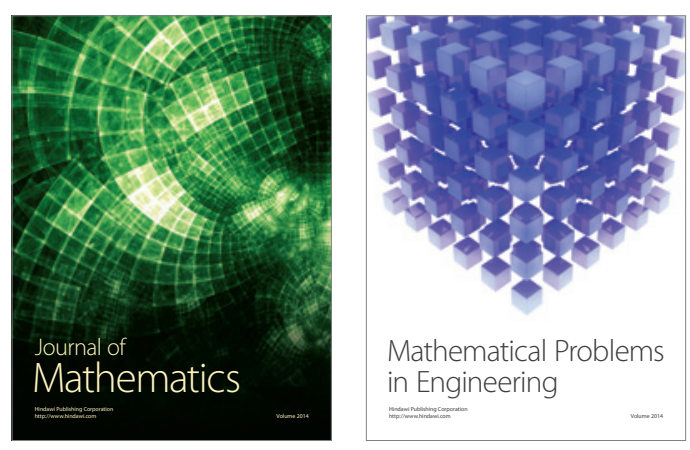

Mathematical Problems in Engineering
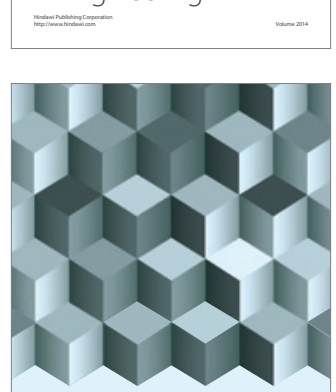

Journal of

Function Spaces
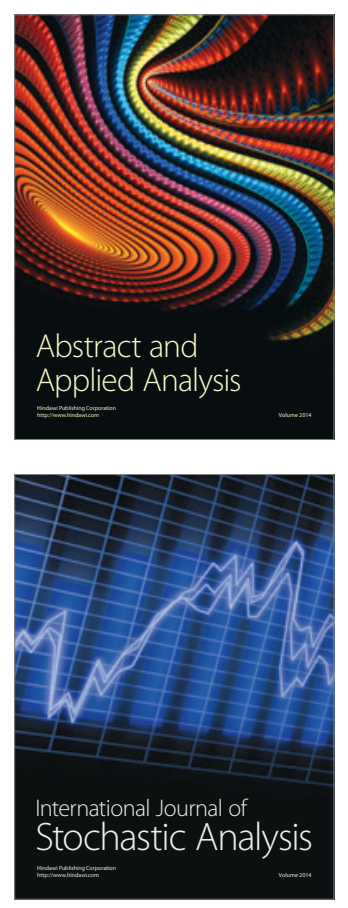

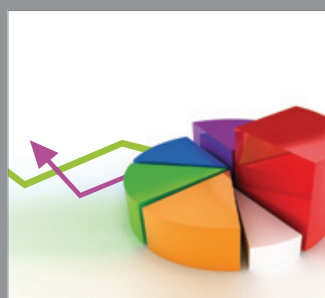

ournal of

Probability and Statistics

Promensencen
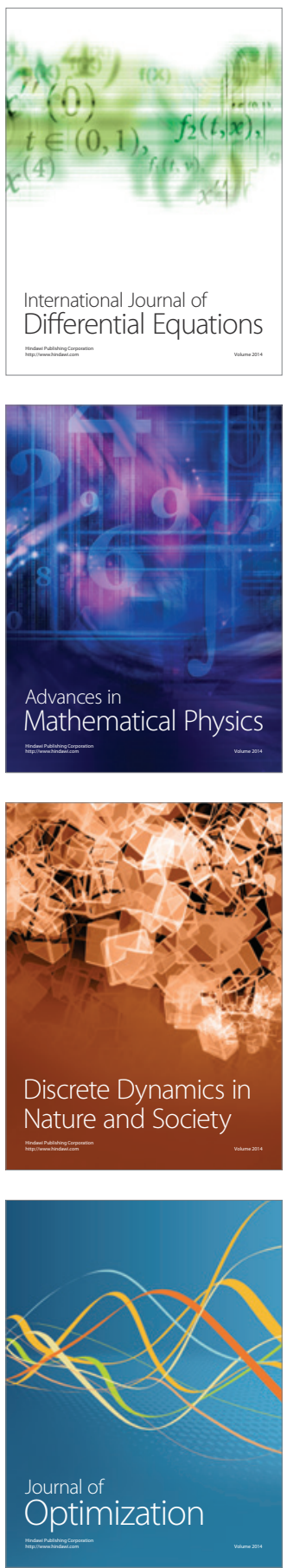